\section{Men of Vision}

The Nobel prize for medicine for 1967 is shared by Professor R. A. Granit, of the Karolinska Institute, Stockholm, Professor H. K. Hartline, of the Rockefeller Institute, New York, and Professor G. Wald, of Harvard University, Massachusetts, the feature common to their work being the analysis of the mechanism of vision.

Granit's analysis of the relatively slow electrical changes that take place when the eye is exposed to light-the electroretinogram, or E.R:G.-was the work that first brought him fame in the field of visual physiology. As with the electrocardiogram, the E.R.G. could be resolved into a number of components, so that the electrical events recorded from the cornea were the algebraic sum of both positive and negative variations.

Subsequent work has largely confirmed his basic postulates, though more precise and localized recording has revealed many instructive complications. From the E.R.G. Granit progressed to the study of the action potentials in single fibres of the optic nerve, studies that culminated in his "dominator-modulator" theory of colour vision. Thus certain fibres appeared to be carrying messages indicating the general luminosity of the stimulus while others, the modulators, appeared to indicate its chromatic quality. The modulators tended to group into three regions of the spectrum, and thus gave an electrophysiological basis to the Young-Helmholtz trichromatic theory. Granit's great achievements in electrophysiology spring from his realization that the retina is, indeed, merely an outgrowth of the forebrain, so that the properties of the synapses within the retina, and the organization of its neurones, are by no means peculiar to this sensory system. His analyses of retinal electrophysiology were thus generalized, and nowhere is this better seen than in his classical book Receptors and Sensory Perception, published in 1955. Furthermore, this realization led him to expand his field of investigation so that the electrophysiology of the proprioceptors of muscles has now become his field of main interest. Thanks largely to Granit's studies and those of younger men from all over the world who gained their early experience in his laboratory, the importance of the spindle in the control of muscular contraction is being realized, while the complex control exerted on muscular action through the spindle by cerebellar and other motor centres is being elucidated.

Hartline, like Granit, made the vertebrate E.R.G. one of his earliest studies in retinal physiology, but very soon moved to a more simply organized eye-namely, that of the horseshoe crab, Limulus. This, like the insect eye, consists of an aggregate of " micro-eyes" or ommatidia, each with its own refracting apparatus, photosensitive cells, and sensory nerve cell. Study of a single ommatidium, with the single group of fibres emerging from it and running to the central nervous system, provided an opportunity to study clearly the electrical events following illumination. A single fibre of the optic nerve of the vertebrate eye is the axon of a ganglion cell of the retina-that is, of a second-order sensory neurone-but the fibre leading from the ommatidium is the axon of a primary sensory neurone (the eccentric cell), and thus the message should be much simpler. The results of Hartline's studies on this system, carried out with ever-improving techniques, form the solid foundation for present-day theories of sensory processes. Thus the concept of the "generator potential," the slow non-propagated negative variation developing in the receptor cell, has received ample justification from his records obtained with micro-electrodes inserted into individual eccentric cells.

Perhaps one of the most interesting discoveries was the mutual inhibition between ommatidia. For a long time if was considered that the responses in fibres emerging from adjacent ommatidia were independent of each other, since there was no clear-cut synapse on the visual pathway where interaction could occur. In fact, however, interaction has been detected, and is thought to occur in the plexus of fibres just outside the ommatidia. It probably represents a crude form of synaptic action, but this awaits elucidation. The importance of this particular aspect is the opportunity it gives to study quantitative aspects of inhibition, since it is becoming clearer, as the study of sensory physiology is pressed further forwards to the sensory and integrating areas of the cerebral cortex, that inhibition is far more important than excitation. Thus uniform illumination of the retina, which excites presumably every rod and cone, produces little or no response in the vast majority of neurones in the visual cortex. Only when the retina is excited by certain patterns of light and dark will these cortical neurones respond ; and the mechanism by which this discrimination is achieved is by way of inhibitory processes taking place at successive levels of the ascending pathway.

Wald has devoted his energies to the elucidation of the chemical events taking place in the receptor following exposure to light and the reversal of these effects during darkadaptation. The amounts of material available for analysis that can be obtained from a single retina are very small, so that special methods must be used to identify them. Wald's initial achievement, which brought him world-wide recognition, was the demonstration that one of the later products of decomposition is retinene, which proved to be a simple derivative of vitamin $\mathrm{A}$, thus providing, incidentally, a rationale of the long-recognized relation between nightblindness and vitamin-A deficiency. Thus rhodopsin, the photopigment of the rods of most vertebrates, could be described as a protein molecule, opsin, with a prosthetic group, retinene. Subsequent studies showed the successive steps in the breakdown of the rhodopsin molecule that culminated in the splitting off of retinene and its conversion to vitamin A in the retina during exposure to light. These changes were "dark reactions" in the sense that, once the first change had taken place in response to light, the succeeding ones occurred on their own; they were the result of thermal, rather than photochemical, reactions. The question that interested the physiologist was the nature of the initial photochemical event that triggered off these later reactions, since it seemed likely that the electrophysiological event would be most closely related to this. Wald showed that one of the earliest events was the "change in shape" of the retinene molecule while it was still attached to the protein, opsin. The retinene molecule, by virtue of having several conjugated double bonds, could adopt a variety of cis-trans-isomeric forms ; and it was shown that only when the molecule had two of these forms could it remain in stable association with the opsin molecule. In the so-called 11-cis-form it united to give the photopigment rhodopsin, while in the 9-cis-form it gave another pigment, isorhodopsin. It would seem, then, that the primary effect of light is to make the linkage between retinene and opsin " uncomfortable," in the sense that the retinene is now no longer able to fit snugly into the opsin carrier. Because of this, the opsin may undergo some strain, and this strain leads to electrical changes, from which changes follow in the neurone associated with the rod-that is, in the bipolar 
cell. Thus Wald's analysis of the chemical events resulting from the impingement of a quantum of light in the rod has carried us very close indeed to the recognition of the real nature of the photochemical stimulus.

These studies have been confined to the photopigments concerned with night vision-namely, rhodopsin, found in the rods of the great majority of vertebrates, and porphyropsin, found in the rods of fresh-water fishes. The two pigments mediate night vision and are apparently not concerned in the more complex daylight, or cone, vision, by virtue of which not only is light appreciated as such but also its qualitative variations are appreciated as colours. In recent years the investigation of the nature of the photopigments in the receptors concerned with daylight and colour vision, the cones, has been pursued in many laboratories. Wald long ago extracted a pigment from the chicken retina, which contains mainly cones, which he called iodopsin. It was entirely different in its light-absorption characteristics from rhodopsin, but like rhodopsin it contained retinene, and it underwent a similar series of chemical changes on illumination. This was only one pigment, however, and the trichromatic theory of colour vision demanded three pigments, contained in separate cones, as the basis for colour discrimination. It would seem that the methods of extraction that were adequate for the study of night-vision pigments were inadequate for extraction and separation of these three hypothetical pigments. And we owe to W. A. H. Rushton, of Cambridge, and R. A. Weale, of London, the detection of several daylight pigments in the mammalian retina. Their studies indicated the presence of red- and green-sensitive pigments; the presence of a bluesensitive pigment was not demonstrated, probably because of the technical difficulties. The Japanese workers Hanoaka and Fujimoto measured the absorption of light by single fish cones, and found characteristic differences corresponding to what might well have been red-, green-, and blue-sensitive cones ; fish cones were chosen because they were big enough to permit this type of measurement. Essentially similar results have been reported in the primate from the laboratories of Marks and of Wald, blue-, green-, and red-sensitive pigments having been described by Wald, for example. Thus the trichromatic theory of Young and Helmholtz has been unequivocally substantiated by modern physiological research.

\section{Fall-out from Space Travel}

The Russians' safe landing of a space ship on Venus is surely one of the most astonishing technical feats so far achieved by man. The skill and resources that have been devoted to it are of necessity immense. While the ultimate goal of space travel still remains a fairly remote possibility, enthusiasts for it, when challenged to justify the vast sums of money they manage to extract from governments, are apt to shelter behind something called "fall-out." This is the expression used when an invention or discovery made for one purpose turns out unexpectedly useful for a different purpose. Something spills over the side of the bandwagon, and a watchful backroom boy rushes in to catch it as it falls.

Many items of space fall-out are listed by Albert $\mathrm{H}$. Schwichtenberg in a recent article ${ }^{1}$. one of three forming an "Aerospace Medical Symposium" in the Fournal of the American Medical Association. Unlike a room or a factory, a spacecraft cabin is hermetically sealed; moreover, airhnrne particles cannot "fall" to the floor. So contamination is far less tolerated. Consequently, methods of reducing it have been developed which can be beneficially applied to industry.

Violent oscillations can arise during take-off of a spacecraft under power, so in the interests of astronauts the frequencies which set up resonance in various parts of the body have had to be investigated. The results are applicable also to motor vehicles and industrial machinery. "Instrument packages" for monitoring the various physiological systems of astronauts have been developed to a high pitch never before attained, and Schwichtenberg mentions a bolometer, fixed near the carotid artery in the neighbourhood of the ear drum, which records minute changes of temperature to an accuracy between $1 / 100$ and $1 / 1,000 \mathrm{C}^{\circ}$. Research already done on the effects of radiation and on shielding and protective drugs could, the author points out, be useful in connexion with nuclear weapons. But it is to be hoped that this particular item of fall-out will be denied the opportunity to justify itself.

Research into weightlessness, which cannot be experienced on earth for more than a fraction of a minute at a time, would hardly be expected to provide fall-out. Yet it belies its name, for fall-out there is. So anxious are the space medicine men to establish the effects of weightlessness over far longer periods than astronauts have yet had to endure that they have experimented with the next best thing-almost total immersion in water. A notable fall-out from this research is the "dry water immersion bed," used for the treatment of intractable trophic ulcers.

So far 19 astronauts have spent a total of some 2,000 weightless hours in 25 flights, writes Charles A. Berry. ${ }^{2}$ The longest flights have lasted 4,8 , and 14 days, and in these the blood of the pilots in command has shown deficits in red cell mass of $5 \%, 20 \%$, and $19 \%$ respectively. ${ }^{3}$ However, this phenomenon, due to a haemolytic process, was not attributed to weightlessness but to the cabin atmosphere being $100 \%$ oxygen. The reason for the pure oxygen is that air pressure inside the cabin is kept at only one-third of the atmospheric pressure on the earth's surface, and the reason for this, in turn, is that experience with pressurized aircraft convinced the engineers that a higher internal pressure would be difficult to maintain.

The forecast, made before space flight actually began, that an atmosphere of $100 \%$ oxygen at low pressure would be harmful was only one of several that proved too pessimistic, and Berry mentions many others. The suits of the "spacewalkers," for instance, showed no sign of the impact of micrometeorites while they floated outside the ship, and still less of any puncture of the suits, inside which a pressure of a quarter of an atmosphere was maintained. But the first space-walker encountered unexpected difficulty which had not been foreseen even by the pessimists: his attempts to complete his " tasks" led to excessive heat and sweat, and he had to hurry back inside. Berry attributes this trouble to the combined effect of "the basic laws of physics producing action and reaction and the need to stop an action, the difficulty in trying to maintain position to do a task, the problems of mobility in a $3.5 \mathrm{lb} . / \mathrm{sq}$. in. (242 millibars) suit, some amount of heat

\footnotetext{
Schwichtenberg, A. H., f. Amer. med. Ass., 1967, 201, 247

2 Berry, C. A., ibid., 1967, 201, 232.

- See Brit. med. 7., 1967, 3, 388.

- Kidera, G. J., f. Amer. med. Ass., 1967, 201, 242.
} 